\title{
Mapping foreign residents in Japan's major cities
}

\author{
Yoshitaka Ishikawa \\ yishikawa@main.teikyo-u.ac.jp, Teikyo University,Tokyo, Japan
}

Keywords: Foreign residents, Ethnic enclaves, Replacement migration

\begin{abstract}
:
Japan's population has been declining since it reached its peak in 2008, and the annual decrease is expected to expand gradually in the future. In this situation, improvement in the fertility rate and intake of foreigners have attracted great attention. However, since the total fertility rate (TFR) has remained stagnant between 1.40 and 1.45 in recent years, more attention is being paid to the role of foreigners as "replacement migration," and the importance of this has even been highlighted by the United Nations (2001). Noteworthy trends in relation to this are the regional disparity of population decline and the distribution of foreign nationals in contemporary Japan. In particular, the issue of depopulation is serious in peripheral parts of the country because it has been occurring since the 1980s. Meanwhile, the monopolar concentration of population, including foreign residents, into Tokyo has also been a major issue.
\end{abstract}

Keeping the above developments in mind, the first edition of Mapping Foreign Residents in Japan, which I edited, was published in 2011; thanks to its favourable reception, a revised edition was published in January 2019. Both editions were prepared especially for policy-makers of national and local government offices in charge of foreign residents. It includes more than 100 maps. This presentation aims to introduce the maps that visualize the status of foreign residents in Japan's major cities and to outline the distinctive features of the differentiation in their residential distribution.

The work's chief sources are national government statistics such as population census (including microdata of foreign residents in the 2015 census), statistics on foreign residents, and vital statistics. The maps are arranged by six topics (distribution/changes, gender/age/nationality, status-of-residence, employment, life, and enclaves/migration), and each topic has several sub-topics. Maps of foreign residents in major cities are drawn on a municipality basis (city, ward, town, and village) or on a small-area unit basis (cho-cho and $a z a$ ). Figure 1 shows an example from the second edition.

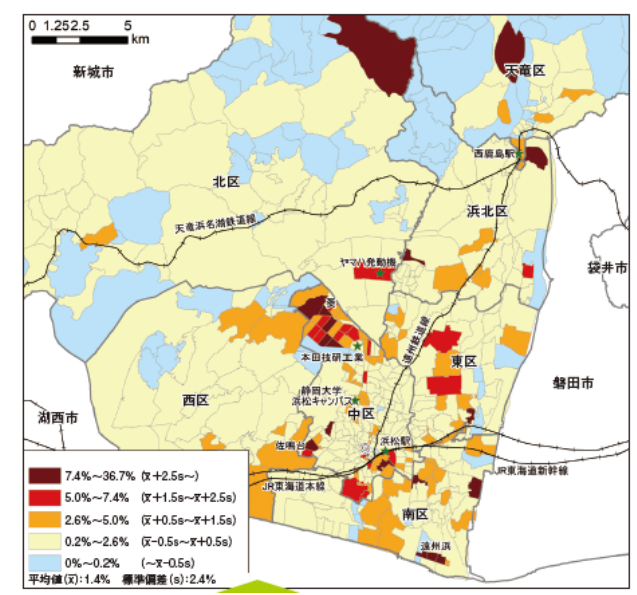

Figure 1. Ratio of foreign population in Hamamatsu City

Major findings obtained from the series of maps in the recently revised publication are summarized as follows.

First, there are very few remarkably large enclaves composed of small-unit areas with a high ratio of foreign residents, suggesting that most foreign residents, even in such areas, tend to reside alongside Japanese residents. Second, major enclaves for 'oldcomers' (Korean and Chinese residents) are located in the inner-city areas of a few metropolises. Third, major enclaves for newcomers are confirmed to exist in four types of small-area units: downtown, public housing, around universities, and around factories. 